\title{
SKETCH OF THE THEORY OF GROWTH OF HOLOMORPHIC FUNCTIONS IN A MULTIDIMENSIONAL TORUS
}

\author{
M. N. Zavyalov and L. S. Maergoiz
}

\begin{abstract}
We develop an approach to the theory of growth of the class $H\left(\mathbb{T}^{n}\right)$ of holomorphic functions in a multidimensional torus $\mathbb{T}^{n}$ based on the structure of elements of this class and well-known results of the heory of growth of entire functions of several complex variables. This approach is illustrated in the case where the growth of the function $g \in H\left(\mathbb{T}^{n}\right)$ is compared with the growth of its maximum modulus on the skeleton of the polydisk. The properties of the corresponding characteristics of growth of the functions in the class $H\left(\mathbb{T}^{n}\right)$ are studied with their relation to coefficients of the corresponding Laurent series. A comparative analysis of these results and similar assertions of the theory of growth of entire functions of several variables is given.
\end{abstract}

Keywords and phrases: entire function of several variables, holomorphic function in multidimensional torus, convex function, characteristics of growth, multiple Laurent series, carrier, strictly convex cone.

AMS Subject Classification: 32A15, 30C45

1. Introduction. In $[7,8]$ we considered the class $H\left(\mathbb{T}^{n}\right)$ of holomorphic functions in the multidimensional torus $\mathbb{T}^{n}=\mathbb{C}_{*}^{n}$, which is the extension of the class $H\left(\mathbb{C}^{n}\right)$ of entire functions of $n$ complex variables. It contains a proper subclass $\mathcal{A}\left(\mathbb{T}^{n}\right)$ consisting of functions that are equivalent to entire functions in the following sense: a function $g$ belongs to the class $\mathcal{A}\left(\mathbb{T}^{n}\right)$ if there exists a monomial holomorphic mapping $\mathcal{F}$ such that $f=g \circ \mathcal{F}$ is an entire function. The interest in these classes is due to the modern research on the analysis of toric varieties (see $[1,2])$.

The theory of entire functions of several variables is well developed (see, e.g., $[3,12,13])$. The aim of this paper is to give an impetus to the development of the theory of growth of holomorphic functions in multidimensional torus. The structure of functions of the class $H\left(\mathbb{T}^{n}\right)$ was studied in $[7,8]$, where indicators of their growth were considered. In [9], an approach was presented to the development of theory of growth of functions of the class $\mathcal{A}\left(\mathbb{T}^{n}\right)$ by the example of studying the properties of their Laplace-Borel transformation based on the corresponding results in the case of entire functions.

In this paper, we study the growth of functions in $H\left(\mathbb{T}^{n}\right)$ in the case where the growth of a function $g \in H\left(\mathbb{T}^{n}\right)$ is compared with the growth of its maximum modulus

$$
M_{g}(r)=\max \left\{|g(z)|:\left|z_{k}\right|=r_{k}, k=1, \ldots, n\right\}, \quad r \in \mathbb{R}_{0}^{n}, \mathbb{R}_{0}=\{r \in \mathbb{R}: r>0\},
$$

on the skeleton of the polydisk. We rely on the known results on the theory of growth in the class $H\left(\mathbb{C}^{n}\right)$ (see [6]). In particular, we study the relationship between the growth and the coefficients of the function $g$ in its Laurent series. Evidence of the obtained statements is given only when it significantly differs from the proof of similar facts in the case of the class $H\left(\mathbb{C}^{n}\right)$.

2. Functions of the class $H\left(\mathbb{T}^{n}\right)$ that are equivalent to entire functions. A natural analog of entire functions of one variable are functions analytic on the Riemann sphere, except for one point. A similar analog in the case of several variables cannot be expected: analytic functions of many variables do not have isolated singularities. The "layout" of the multidimensional analog of this result is suggested by the following version of the Hadamard-Valiron theorem. 
Theorem 2.1. Let $g(z)=g\left(z_{1}, \ldots, z_{n}\right)$ be a function that is holomorphic in the torus $\mathbb{T}^{n}, n>1$; $M_{g}(r)$ be its maximum modulus on the skeleton of the polydisk (see (1)). Then both $M_{g}(r)$ and $\ln M_{g}(r)$ are convex functions of $\ln r_{1}, \ldots, \ln r_{n}$.

In the case where $g$ is the trace of an entire function on $\mathbb{T}^{n}, M_{g}(r)$ is an increasing function with respect to each variable. Hence

$$
V_{g}(u)=\ln M_{g}\left(e^{u}\right):=\ln M_{g}\left(e^{u_{1}}, \ldots, e^{u_{n}}\right), \quad u \in \mathbb{R}^{n},
$$

is a convex function with a convex cone $K_{V}$ of decrease directions, and $K_{V}$ contains $\mathbb{R}_{-}^{n} \backslash 0$, where $\mathbb{R}_{-}=\{u \in \mathbb{R}: u \leq 0\}$.

2.1. Convex functions equivalent to increasing functions. For a rigorous definition of the cone $K_{V}$, we need the following facts of convex analysis.

Theorem 2.2 (see [11, Theorem 8.5]). Let $V=V(u), u \in \mathbb{R}^{n}$ be a finite convex function. For any $a \in \mathbb{R}^{n}$, there exists the limit

$$
\gamma_{V}(u)=\lim _{t \rightarrow \infty} \frac{V(t u+a)-V(a)}{t}=\sup _{t>0}\left\{\frac{V(t u+a)-V(a)}{t}\right\} \quad \forall u \in \mathbb{R}^{n}
$$

which is independent of a. In addition, the function $\gamma_{V}$ is a positive-homogeneous convex function, which takes, possibly, a value of $+\infty$.

The function $\gamma_{V}$ is called the asymptotic (or recessive) function of $V$. In [11, Sec. 8], this definition is given in an equivalent form (see [6, Chap. 1, Sec. 6]).

Theorem 2.3 (see [11, Theorem 8.6, Corollary 8.6.1]). The trace $\varphi(t)=V(u t+a), t \in \mathbb{R}$, of a convex function $V(x), x \in \mathbb{R}^{n}, n>1$, on the line $\Gamma(u, a)=\{u t+a, t \in \mathbb{R}\}$, where $u \in \mathbb{R}^{n} \backslash\{0\}$, a $\in \mathbb{R}^{n}$, is a nonconstant decreasing function if and only if the condition $\gamma_{V}(u) \leq 0, \gamma_{V}(-u)>0$ is satisfied.

In particular, if we have $\gamma_{V}(u)=\gamma_{V}(-u)=0$ for given $u \in \mathbb{R}^{n} \backslash\{0\}$, then the function $V$ is constant on the line $\Gamma(u, a)$. This result stimulated the following definition.

Definition 2.1 (see [7] and [8, Definition 1.6]). Let $V(u), u \in \mathbb{R}^{n}, n>1$, be a convex function, and $\gamma_{V}$ be its asymptotic function. The set

$$
K_{V}=\left\{u \in \mathbb{R}^{n}: \gamma_{V}(u) \leq 0\right\}
$$

is called the cone of decrease directions of the function $V$.

Now we state the criterion of equivalence of a given convex function to a convex function increasing in each variable (a generalization of Theorem 1.9 in $[7,8]$ ). For this we need the following assertion.

Lemma 2.1. Under the conditions and notation of Definition 2.1, the set

$$
L_{V}=\left\{u \in K_{V}: \gamma_{V}(u)=\gamma_{V}(-u)=0\right\}
$$

is a linear subspace of $\mathbb{R}^{n}$, and $V(u) \equiv V(0)$ for all $u \in L_{V}$.

Proof. 1. Take $u \in L_{V}, \tau \in \mathbb{R}$. From the property of positive homogeneity of the function $\gamma_{V}$ we have $\tau u \in L_{V}$ (see Theorem 2.2 and (5)). Since, in addition, $\gamma_{V}$ is a convex function, we have

$$
\left.\gamma_{V}[ \pm(x+y)] \leq \gamma_{V}( \pm x)\right]+\gamma_{V}( \pm y)=0 \quad \forall x, y \in L_{V}
$$

On the other hand,

$$
0=\gamma_{V}(0) \leq \gamma_{V}(x+y)+\gamma_{V}[(-x-y)] \quad \forall x, y \in L_{V} .
$$

This implies that $\gamma_{V}(x+y)=\gamma_{V}[(-x-y)]=0$, i.e., $x+y \in L_{V}$. Therefore, $L_{V}$ is a linear subspace $\mathbb{R}^{n}$. 
For $a=0$, from the formula (3) we conclude that

$$
V(u t) \leq V(0)+\gamma_{V}(u t) \quad \forall t \geq 0, u \in \mathbb{R}^{n} .
$$

Hence, $V(u) \leq V(0)$ for all $u \in L_{V}$ (see (5)). Since the function $V$ is convex and bounded from above on $L_{V}$, it is constant on $L_{V}$ (see [11, Corollary 8.6.2]).

Theorem 2.4. Let $V(x), x \in \mathbb{R}^{n}$ be a nonconstant convex function, $\gamma_{V}$ be its asymptotic function, $K_{V}$ be the cone of decrease directions of $V$, and $L_{V}$ be a subset of $K_{V}$ (see (3)-(5)). The equalities $\operatorname{dim} K_{V}=n$ and $\operatorname{dim} L_{V}=m$, where $0 \leq m<n$, are satisfied if and only if there exists a nondegenerate linear mapping $A: \mathbb{R}^{n} \rightarrow \mathbb{R}^{n}, u=A v$, possessing the following property: $W(v):=V(A v), v \in \mathbb{R}^{n}$, is a convex function increasing in each of the variables $v_{1}, \ldots, v_{p}$, where $p=n-m$, and, under the condition $m>0$, is independent of the remaining variables $v_{p+1}, \ldots, v_{n}$.

Proof. Necessity. Assume that $m>0$. By the condition of the theorem, $V \neq$ const and $\operatorname{dim} K_{V}=n$. Therefore, the representation

$$
\mathbb{R}^{n}=L_{V}^{\perp} \oplus L_{V}, \quad K_{V}=\mathcal{K}_{V} \oplus L_{V},
$$

is valid and $\operatorname{dim} L_{V}^{\perp}=\operatorname{dim} \mathcal{K}_{V}=p>0$. Note that $L_{V}=K_{V} \cap\left(-K_{V}\right)$ is the maximum linear subspace contained in $K_{V}$ (see [11, Theorem 2.7]). Hence, $\mathcal{K}_{V}$ is a strictly convex cone (i.e., a cone that does not contain straight lines). We choose a basis $b^{(k)} \in K_{V}, k=1, \ldots, n$, such that in the notation used in the formulas (4) and (5), the following condition holds:

$$
b^{(k)} \in \mathcal{K}_{V} \subset L_{V}^{\perp}, \quad k=1, \ldots, p ; \quad b^{(k)} \in L_{V}, \quad k=p+1, \ldots, n .
$$

We define a linear mapping $D: \mathbb{R}^{n} \rightarrow \mathbb{R}^{n}$ with by the conditions $D\left[b^{(k)}\right]=-e_{k}, k=1, \ldots, n$, where $\left\{e_{k}\right\}_{1}^{n}$ is the standard basis in $\mathbb{R}^{n}$. It possesses the property $D\left(I_{V}\right)=\mathbb{R}_{-}^{n}$, where $I_{V} \subset K_{V}$ is the convex cone with vertex at 0 generated by the $n$-dimensional simplex with vertices $\left\{0,\left\{b_{k}\right\}_{1}^{n}\right\}$ and $\mathbb{R}_{-}^{n}$ is the negative octant in $\mathbb{R}^{n}$. In addition, $D\left(L_{V}\right)=\mathbb{R}^{m}$ is the linear span of the vectors $\left\{e_{k}, k=p+1, \ldots, n\right\}$ since, due to Lemma 2.1, $L_{V}$ is a linear subspace $\mathbb{R}^{n}$. This and Lemma 2.1 imply that the desired mapping is $A=D^{-1}$. In the case $m=0$, the proof is much simpler.

Sufficiency is proved by the same arguments in the reverse order.

Thus, a convex function $V$ given in $\mathbb{R}^{n}$ is "equivalent" to a convex function $W$, which increases in each variable, under the condition $\operatorname{dim} K_{V}=n$; moreover, $W$ may depend on less number of variables. In the case where, in the notation used in the formula (2), $V=V_{g}$ for the function $g$ from a certain subclass of holomorphic functions in $\mathbb{T}^{n}, n>1$, all coefficients of the mapping $A$ can be chosen to be integers.

2.2. Structure of functions that are equivalent to entire functions and their properties. Consider the proper subclass $\mathcal{A}\left(\mathbb{T}^{n}\right)$ of the class $H\left(\mathbb{T}^{n}\right)$ consisting of holomorphic functions in the space $\mathbb{T}^{n}$, which are equivalent to entire functions in the following sense.

Definition 2.2 (see [7] and [8, Definition 3.1]). We say that a function $g \in H\left(\mathbb{T}^{n}\right), n>1$, is equivalent to an entire function $f$ if there exists a monomial mapping

$$
\mathcal{F}=\mathcal{F}_{g}: \mathbb{T}^{n} \rightarrow \mathbb{T}^{n} ; \quad z=\mathcal{F}(w), \quad z_{k}=\prod_{j=1}^{n} w_{j}^{s_{k j}}, \quad k=1, \ldots, n,
$$

where $B:=\left\|s_{k j}\right\|$ is an integer nondegenerate square $(n \times n)$-matrix such that the function $f(w)=$ $[g \circ \mathcal{F}](w)$ admits an analytic continuation $F(w)$ to $\mathbb{C}^{p}$, where $p \leq n$. This means that $F$ is an entire function of $p$ complex variables $w_{1}, \ldots, w_{p}$, which is independent ${ }^{1}$ of $w_{p+1}, \ldots, w_{n}$ for $p<n$. In this case, we write $g \sim f$.

\footnotetext{
${ }^{1}$ This can be assumed without loss of generality.
} 
Remark 2.1 (see [9, Sec. 1]). The inverse mapping $\mathfrak{F}^{-l}$ is a monomial mapping with fractional exponents, i.e., it is multivalued in $\mathbb{T}^{n}$. However, $g_{1}(z):=\left[f \circ \mathcal{F}^{-1}\right](z), z \in \mathbb{T}^{n}$, is a single-valued function such that $g_{1}(z) \equiv g(z), z \in \mathbb{T}^{n}$. In order to verify this, we need the notion of the supports of the Laurent series

$$
g(z)=\sum_{k \in \mathbb{Z}^{n}} a_{k} z^{k}, \quad z \in \mathbb{T}^{n} ; \quad z^{k}=z_{1}^{k_{1}} \ldots z_{n}^{k_{n}}
$$

for the function $g$ and the power series

$$
f(w)=[g \circ \mathcal{F}](w)=\sum_{m \in \mathbb{Z}_{+}^{p}} c_{m} w^{m}, \quad w \in \mathbb{T}^{p},
$$

converging to an entire function. These sets are defined as follows:

$$
S_{g}=\left\{k \in \mathbb{Z}^{n}: a_{k} \neq 0\right\}, \quad S_{f}=\left\{m \in \mathbb{Z}_{+}^{p}: c_{m} \neq 0\right\} .
$$

Comparing coefficients of these series, we obtain the relations

$$
S_{f}=B^{\prime}\left[S_{g}\right], \quad c_{m}=a_{k}, \quad m=B^{\prime} k \in S_{f}, \quad k \in S_{g},
$$

where $B^{\prime}$ is the transposed matrix for $B=\left\|s_{k j}\right\|$ (see (6)). It is convenient to assume that $S_{f} \subset$ $\mathbb{Z}_{+}^{n}$, since in Definition 2.2 the condition on the dependence of the function $f$ only on the variables $w_{1}, \ldots, w_{p}$ for $p<n$ means that $m_{p+1}=\cdots=m_{n}=0$ for all $m \in S_{f}$. In this notation, the following expansion holds:

$$
g_{1}(z)=\left[f \circ \mathcal{F}^{-1}\right](z)=\sum_{m \in S_{f}} c_{m}\left[\mathcal{F}^{-1}(z)\right]^{m}, \quad z \in \mathbb{T}^{n} .
$$

From the relations for the supports $S_{f}$ and $S_{g}$ we deduce

$$
\left\langle m, B^{-1}(\ln z)\right\rangle=\left\langle B^{\prime} k, B^{-1}(\ln z)\right\rangle=\langle k, \ln z\rangle, \quad k=\left(B^{\prime}\right)^{-1} m \in S_{g}, \quad m \in S_{f} ; \quad z \in \mathbb{T}^{n} .
$$

Here and below, $\langle\cdot, \cdot\rangle$ is the scalar product in $\mathbb{C}^{n}$. This and the relations for the coefficients of the series for $f$ and $g$ imply the following:

$$
c_{m}\left[\mathcal{F}^{-1}(z)\right]^{m}=a_{k} z^{k}, \quad k=\left(B^{\prime}\right)^{-1} m \in S_{g}, \quad m \in S_{f} ; \quad z \in \mathbb{T}^{n} .
$$

The equality $g_{1}=g$ follows from these arguments.

The entire function $f=g \circ \mathcal{F}$ is said to be equivalent for a function $g \in \mathcal{A}\left(\mathbb{T}^{n}\right)$.

Example 2.1 (see $\left[8\right.$, example $\left.1^{\prime}\right]$ ). Let $K$ be an obtuse angle with vertex $0 \in \mathbb{R}^{3}$ whose sides have the directing vectors $c_{1}=(1,2,-3)$ and $c_{2}=(-3,-1,4)$ and let $g(z)$ be a holomorphic function of three complex variables in $\mathbb{T}^{3}$ represented by the Laurent series (7) with $n=3$ whose support is $S_{g}=K \cap \mathbb{Z}^{3}$. Based on Definition 2.2, we show that $g \in \mathcal{A}\left(\mathbb{T}^{3}\right)$.

It is easy to see that the angle $K$ is located in the plane $k_{1}+k_{2}+k_{3}=0, k \in \mathbb{R}^{3}$. Therefore, in this case, the Laurent series of $g$ in (7) admits the representation

$$
g(z)=\sum_{k \in S_{g}} a_{k}\left(\frac{z_{1}}{z_{3}}\right)^{k_{1}} \cdot\left(\frac{z_{2}}{z_{3}}\right)^{k_{2}}, \quad z \in \mathbb{T}^{3} ; \quad k_{3}=-k_{1}-k_{2} .
$$

Consider the mapping

$$
z=\mathcal{F} w, \quad w \in \mathbb{T}^{3}, \quad z_{1}=\frac{w_{3}}{w_{1} w_{2}^{2}}, \quad z_{2}=w_{1}^{3} w_{2} w_{3}, \quad z_{3}=w_{3}
$$

and the series

$$
f(w)=[g \circ \mathcal{F}](w)=\sum_{m \in S_{f}} b_{m} w_{1}^{m_{1}} w_{2}^{m_{2}}, \quad b_{m}=a_{k}, \quad m_{1}=-k_{1}+3 k_{2}, \quad m_{2}=-2 k_{1}+k_{2}, \quad m_{3}=0 .
$$


Here $k \in S_{g} \subset K$, with $k_{3}=-k_{1}-k_{2}$, and $\left(k_{1}, k_{2}\right) \in \hat{K}$, where $\hat{K}$ is the projection of the angle $K$ on $\mathbb{R}^{2}$. Hence we deduce $\left(k_{1}, k_{2}\right)=m_{1} h_{1}+m_{2} h_{2}, m \in S_{f}$, where $h_{1}=(1,2) / 5$ and $h_{2}=(-3,-1) / 5$ are the directing vectors of the sides of the angle $\hat{K}$. From elementary geometric fact we obtain that $m_{i} \geq 0, i=1,2$, i.e., for any element $m$ of the support $S_{f}$ we have $\left(m_{1}, m_{2}\right) \in \mathbb{Z}_{+}^{2}$. Thus, $g \sim f$.

Theorem 2.4 implies the following property of functions of the class $\mathcal{A}\left(\mathbb{T}^{n}\right), n>1$.

Corrolary 2.1. Let $g \in \mathcal{A}\left(\mathbb{T}^{n}\right)$ and $M_{g}(r)$ be its maximum modulus on the skeleton of the polydisk,

$$
V(u):=V_{g}(u)=\ln M_{g}\left(e^{u}\right), \quad u \in \mathbb{R}^{n}
$$

(see Definition 2.2 and (1)-(2)). Then, in the notation of the formulas (4) and (5), the following equalities hold:

$$
\operatorname{dim} K_{V}=n, \quad \operatorname{dim} L_{V}=m:=n-p,
$$

where $0 \leq m<n$.

Proof. Let $f$ be an entire function equivalent to $g$. According to Definition 2.2 (see (6)), there is a mapping

$$
E: \mathbb{R}_{0}^{n} \rightarrow \mathbb{R}_{0}^{n} ; \quad r=E(q), \quad r_{k}=\prod_{j=1}^{n} q_{j}^{s_{k j}}, \quad k=1, \ldots, n,
$$

where $E$ is the trace of the mapping $\mathcal{F}$ on $\mathbb{R}_{0}^{n}, B=\left\|s_{k j}\right\|$ is an integer, nondegenerate $(n \times n)$-matrix such that $M_{f}(q)=M_{g} \circ E(q), q \in \mathbb{R}_{0}^{n}, M_{f}$ is the maximum modulus of $f$ on the skeleton of the polydisk. From (8) we conclude that the linear mapping $B: \mathbb{R}^{n} \rightarrow \mathbb{R}^{n}, u=B v$, defined by the matrix $B$, possesses the property

$$
V_{f}(v):=\ln M_{f}\left(e^{v}\right)=V_{g}(B v), \quad v \in \mathbb{R}^{n},
$$

and the convex function $V_{f}$ (see Theorem 2.1) increases in each of the variables $v_{1}, \ldots, v_{p}$, and under the condition of $p<n$, it is independent of the remaining variables $v_{p+1}, \ldots, v_{n}$. To complete the proof, it remains to apply Theorem 2.4 .

Based on geometric properties of coefficients of the Laurent series of a function $g$ from the class $H\left(\mathbb{T}^{n}\right)$, we state a criterion of its belonging to the class $\mathcal{A}\left(\mathbb{T}^{n}\right)$.

Theorem 2.5 (see [7] and [8, Theorem 3.2]). Assume that a function $g(z)$ belongs to the class $H\left(\mathbb{T}^{n}\right)$, $n>1$, and let its expansion in a multiple Laurent series of the form (7) be given. Let $K_{g}$ be the smallest closed convex cone with vertex $0 \in \mathbb{R}^{n}$ containing the support $S_{g}$ of the series (7). The function $G$ belongs to the class $\mathcal{A}\left(\mathbb{T}^{n}\right)$ (i.e., in terms of Definition 2.2, $f(w)=[g \circ \mathcal{F}](w)$ is an entire function; see (6)) if and only if the set $K_{g}$ is a strictly convex cone. In addition, the following statements are valid:

(1) the support $S_{f}$ of the power series of the function $f$ belongs to a certain sublattice of $\mathbb{Z}_{+}^{n}$ and possesses the property $S_{f}=B^{\prime}\left[S_{g}\right]$, where $S_{g}$ is the support of the Laurent series (7), and $B^{\prime}$ : $\mathbb{R}^{n} \rightarrow \mathbb{R}^{n}$ is the mapping generated by the matrix transposed to $B=\left\|s_{k j}\right\|$ (see Definition 2.2 and the remark to it);

(2) if $\operatorname{dim} K_{g}=p \leq n$, then $f$ is an entire function of $p$ complex variables.

Functions of the class $\mathcal{A}\left(\mathbb{T}^{n}\right)$ play the key role in the structure of elements of the class $H\left(\mathbb{T}^{n}\right)$. The following multidimensional analog of the Laurent theorem holds.

Theorem 2.6 (see [7] and [8, Theorem 3.3]). Let $g \in H\left(\mathbb{T}^{n}\right)$. Then

$$
g(z)=\sum_{i=1}^{m} g_{i}(z), \quad z \in \mathbb{T}^{n}, \quad m=m(g) \leq n+1,
$$


where $\left\{g_{i}\right\}_{1}^{m} \subset H\left(\mathbb{T}^{n}\right)$ are functions equivalent to entire functions (see Definition 2.2). Moreover, for any function $g_{i}$ its support $S\left(g_{i}\right)$ possesses the property $S\left(g_{i}\right) \subset K_{i}$ for all $i=1, \ldots, m$, where $K_{i}$ is a strictly convex cone in $\mathbb{R}^{n}$ with vertex 0 such that $\operatorname{dim} K_{i}=n$. Moreover,

$$
K_{i} \cap K_{j}=\varnothing, \quad i \neq j, \quad(i, j) \subset\{1, \ldots, m\} .
$$

3. Characteristics of growth of functions of the class $H\left(\mathbb{T}^{n}\right)$ and their properties. In $[7,8]$, by analogy with the growth indices of entire functions of several variables (see [4-6]), the asymptotic growth characteristics of functions of class $H\left(\mathbb{T}^{n}\right)$ are given. Let us recall their definitions, necessary for the further presentation.

3.1. Order function. Let $g \in H\left(\mathbb{T}^{n}\right)$ and $M_{g}$ be the maximum modulus of the function $g$ on the skeleton of the polydisk (see (1)).

Definition 3.1 (see [7], [8, Definition 3.5]). The order function of a function $g \in H\left(\mathbb{T}^{n}\right)$ is the function

$$
\rho_{g}(u)=\limsup _{t \rightarrow \infty} \frac{\ln ^{+} \ln ^{+} M_{g}\left(t^{u}\right)}{\ln t}, \quad t^{u}=t^{u_{1}}, \ldots, t^{u_{n}}, \quad t>0, \quad u \in \mathbb{R}^{n},
$$

where $\ln ^{+} S=\max |\ln S, 1|, S>0$. A function $g$ is called a function of finite order if its order function $\rho_{g}(u), u \in \mathbb{R}^{n}$, is a finite function.

As was noted in [8, Proposition 3.6], the order function $\rho_{g}$ of a function $g \in H\left(\mathbb{T}^{n}\right)$ is a nonnegative sublinear function in $\mathbb{R}^{n}$, possibly not finite everywhere. If $g$ is not an entire function, then $\rho_{g}$ is not a function increasing in each variable; moreover, the cone of decrease directions (see Definition 2.1) can be an empty set. However, the geometric meaning of the order function $\rho_{g}$ for $g \in H\left(\mathbb{T}^{n}\right)$ remains the same as in the case of entire functions. To ascertain this, we need the following notion from convex analysis.

Definition 3.2 (see [11, Sec. 8]). Let $T$ be an unbounded closed convex set in $\mathbb{R}^{n}$ and $\operatorname{dim} T=n$. The asymptotic cone $A(T)$ of the set $T$ is the maximal cone with vertex 0 such that

$$
A(T)=\left\{u \in \mathbb{R}^{n}: T+u \subset T\right\} .
$$

Proposition 3.1 (see [11, Sec. 8], [6, Chap. 1, Theorem 6.5]). Let $V(u), u \in \mathbb{R}^{n}$, be a convex function, and

$$
\text { epi } V=\left\{\left(u, u_{m+1}\right) \in \mathbb{R}^{m} \times \mathbb{R}: u_{m+1} \geq V(u)\right\}
$$

be its epigraph. Then the asymptotic cone $A$ (epi $V$ ) of the epigraph of $V$ coincides with the epigraph epi $\gamma_{V}$ of its asymptotic function $\gamma_{V}($ see Theorem 2.2).

Now we discuss the geometric property of the function $\rho_{g}$ (see (11)). We set

$$
\Phi_{g}(r)=\ln ^{+} M_{g}(r), \quad r \in \mathbb{R}_{0}^{n} ; \quad W_{g}(u)=\ln ^{+} \Phi_{g}\left(e^{u}\right), \quad u \in \mathbb{R}^{n} .
$$

If $W_{g}$ is a convex function, then the function $\rho_{g}$ is its asymptotic function (see (2), Theorems 2.1 and 2.2), and according to Proposition 3.1, the epigraph epi $\rho_{g}$ is the asymptotic cone $A$ (epi $W_{g}$ ) of the epigraph of the function $W_{g}$. A similar property of $\rho_{g}$ is also valid in the general case, where $V:=W_{g}$ is a quasi-convex function satisfying the inequality

$$
V(\lambda x+(1-\lambda) y) \leq \max \{V(x), V(y)\} \quad \forall \lambda \in[0,1], x, y \in \mathbb{R}^{n}
$$

(see [6, Chap. 6, Sec. 2]).

We show that the definition of the order function $\rho_{g}$ for $g \in H\left(\mathbb{T}^{n}\right)$ is independent of the choice of a parabolic ray of a more general form

$$
L(r, u)=\left\{r t^{u}=\left(r_{1} t^{u_{1}}, \ldots, r_{n} t^{u_{n}}\right): t>0\right\}, \quad u \in \mathbb{R}^{n} \backslash\{0\}, \quad r \in \mathbb{R}_{0}^{n},
$$


as in the case of entire functions (see [6, Chap. 6, lemma 2.8]) and functions of the class $\mathcal{A}\left(\mathbb{T}^{n}\right)$ (see [7] and $[8$, Theorem 3.9]).

Theorem 3.1. In the notation accepted, for any given $x \in \mathbb{R}^{n} \backslash\{0\}$, the trace of the function $\Phi_{g}=$ $\ln ^{+} M_{g}$ on each parabolic half-ray of $\left\{L(r, x), r \in \mathbb{R}_{0}^{n}\right\}$ (see (13)) has the growth order

$$
\psi_{x}(r)=\limsup _{t \rightarrow \infty} \frac{\ln ^{+} \Phi_{g}\left(r t^{x}\right)}{\ln t} \equiv \rho_{g}(x), \quad r \in \mathbb{R}_{0}^{n},
$$

where $\rho_{g}$ is the order function for $g \in H\left(\mathbb{T}^{n}\right)$.

Proof. If $\psi_{x}(r) \not \equiv \infty$ for $r \in \mathbb{R}_{0}^{n}$, then there exists a point $a \in \mathbb{R}_{0}^{n}$ such that $0 \leq \psi_{x}(a)<\infty$. Take $r \in \mathbb{R}_{0}^{n} \backslash\{a\}$. According to Theorem 2.1, $\Phi_{g}(r)=\ln ^{+} M_{g}(r)$ is a convex function, and $\ln ^{+} \Phi_{g}(r)$ is a quasi-convex function of $\ln r_{1}, \ldots, \ln r_{n}$. Therefore, the following inequality holds:

$$
\Phi_{g}\left(t_{1}^{\lambda} s_{1}^{\mu}, \ldots, t_{n}^{\lambda} s_{n}^{\mu}\right) \leq \max \left\{\Phi_{g}(t), \Phi_{g}(s)\right\} \quad \forall\{t, s\} \in \mathbb{R}_{0}^{n},
$$

where $\lambda \in(0,1), \mu=1-\lambda$. Assuming that $t_{i}=a_{i} \cdot t^{x_{i} / \lambda}$ and $s_{i}=a_{i}^{-\lambda / \mu} \cdot r_{i}^{1 / \mu}, i=1, \ldots, n$, we find from (15) and (14):

$$
\Phi_{g}\left(r t^{x}\right) \leq \max \left\{\Phi_{g}\left(a t^{x / \lambda}\right), A\right\} \leq \max \left\{t^{\varepsilon+\psi_{x}(a) / \lambda}, A\right\} \quad \forall t>t_{0}(\varepsilon),
$$

where $A=\Phi\left(r_{1}^{1 / \mu} \cdot a_{1}^{-\lambda / \mu}, \ldots, r_{n}^{1 / \mu} \cdot a_{n}^{-\lambda / \mu}\right), \varepsilon>0$. Hence we deduce

$$
\psi_{x}(r) \leq \varepsilon+\frac{\psi_{x}(a)}{\lambda}
$$

or (passing to the limit as $\lambda \rightarrow 1$ and $\varepsilon \rightarrow 0$ )

$$
\psi_{x}(r) \leq \psi_{x}(a)
$$

Thus, $\psi_{x}(r)$ is a bounded function in $\mathbb{R}_{0}^{n}$. Changing the places of $a$ and $r$ with each other in these arguments, we conclude that $\psi_{x}(a) \leq \psi_{x}(r)$. Consequently,

$$
\psi_{x}(r) \equiv \psi_{x}(a), \quad r \in \mathbb{R}_{0}^{n}, \quad \rho_{g}(x)=\psi_{x}(\mathbb{I})=\psi_{x}(a) \quad \forall a \in \mathbb{R}_{0}^{n}, \quad \mathbb{I}=(1, \ldots, 1) .
$$

The theorem is proved.

We mention the following criterion for a finite-order function of the class $\mathcal{A}\left(\mathbb{T}^{n}\right)$.

Proposition 3.2 (see [7] and [8, Proposition 3.8]). Assume that a function $g$ belongs to the class $\mathcal{A}\left(\mathbb{T}_{n}\right)$ and $K_{V}$ is the cone of decrease directions of the function $V_{g}(u)=\ln M_{g}\left(e^{u}\right), u \in \mathbb{R}^{n}$ (see Definition 2.1) with vertex at 0 . Assume that $x$ is an arbitrary fixed element of $\mathbb{R}^{n} \backslash\{0\}$ such that $-x \in \operatorname{int} K_{V}$ and $\rho_{g}$ is the order function for $g$. If $\rho_{g}(x)<\infty$, then $g$ is a finite-order function.

The positive homogeneity of the order function $\rho_{g}$ for $g \in H\left(\mathbb{T}^{n}\right)$ implies that its (finite) positive values are determined by the set

$$
T_{g}=\left\{u \in \mathbb{R}^{n}: \rho_{g}(u)=1\right\},
$$

which is called the order hypersurface of the function $g$.

Example 3.1. For $n=1$, the Laurent theorem on the expansion of a holomorphic function in a ring implies that for any function $g \in H(\mathbb{T})$, the following representation is valid:

$$
g(z)=g_{+}(z)+g_{-}\left(\frac{1}{z}\right), \quad z \in \mathbb{T}=\mathbb{C} \backslash\{0\},
$$


where $g_{+}$and $g_{-}$are entire functions. If they have finite nonzero orders $\rho_{+}$and $\rho_{-}$, respectively, then the order function is

$$
\rho_{g}(u)= \begin{cases}\rho_{+} u, & u>0, \\ \rho_{-} u, & u \leq 0\end{cases}
$$

Therefore, the set $T_{g}$ consists of two points $1 / \rho_{+}$and $1 / \rho_{-}$.

3.2. Type function in a given direction of growth. Now let us consider a more subtle indicator of growth of functions from the class $H\left(\mathbb{T}^{n}\right)$.

Definition 3.3 (see [7], [8, Definition 3.10]). Let $g \in H\left(\mathbb{T}^{n}\right)$ and let $\rho_{g}$ be the order function for the function $g$. We assume that $\rho_{g}(x) \in(0, \infty)$ for fixed $x \in \mathbb{R}^{n} \backslash\{0\}$. The function

$$
\sigma_{g}(r ; x)=\limsup _{t \rightarrow \infty} \frac{\ln ^{+} M_{g}\left(r t^{x}\right)}{t^{\rho_{g}(x)}}, \quad r \in \mathbb{R}_{0}^{n},
$$

is called the type function in the direction $x$ or the $x$-type function for the function $g$, and the value $\sigma_{g}(x):=\sigma_{g}(\mathbb{I} ; x)$, where $\mathbb{I}=(1, \ldots, 1)$, is called its type in the direction $x$.

The following formula holds (see [7], [8, remark to Definition 3.10]):

$$
\sigma_{g}(\cdot ; x)=\sigma_{g}(\cdot ; x \tau) \quad \forall \tau>0 .
$$

Therefore, without loss of generality, in the definition of the $x$-type function, it suffices to impose the condition $x \in T_{g}$ (see (17) and (18)). We recall the simplest properties of the $x$-type function (see [7] and [8, Proposition 3.11]).

Proposition 3.3. The functions $\sigma_{g}\left(e^{u} ; x\right)$ and $\delta_{g}^{x}(u):=\ln \sigma_{g}\left(e^{u} ; x\right)$ are convex functions in their domain $D$, and if $x \in T_{g}$, then

$$
\sigma_{g}\left(r t^{x} ; x\right)=t \sigma_{g}(r ; x) \quad \forall r \in \mathbb{R}_{0}^{n}, \quad t>0 ; \quad \delta_{g}^{x}(u+x \tau)=\delta_{g}^{x}(u)+\tau \quad \forall u \in \mathbb{R}^{n}, \quad \tau \in \mathbb{R} .
$$

As in the case of the order function, $\sigma_{g}(\cdot ; x)$ and $\delta_{g}^{x}$ belong to a wider class of functions compared with the situation where $g \in H\left(\mathbb{C}^{n}\right)$ (see Sec. 3.1). Let us show that, in spite of this geometric property of the function $\delta_{g}^{x}$, the requirement $g \in H\left(\mathbb{T}^{n}\right)$ is completely analogous to a similar property in $H\left(\mathbb{C}^{n}\right)$.

For simplicity, in the notation of Proposition 3.3, we set $D=\mathbb{R}^{n}$. From (19) we conclude that the epigraph (see Proposition 3.1) $I_{g}(x):=$ epi $\delta_{g}^{x}$ of the function $\delta_{g}^{x}$ is a convex cylinder. Let $\Phi_{g}=\ln ^{+} M_{g}$, $W_{g}(u)=\ln ^{+} \Phi_{g}\left(e^{u}\right), u \in \mathbb{R}^{n}$. The relations (18) and (19) imply the equality

$$
\limsup _{\tau \rightarrow \infty}\left[W_{g}(x \tau+u)-\tau-\delta_{g}^{x}(u)\right]=\limsup _{\tau \rightarrow \infty}\left[W_{g}(x \tau+u)-\delta_{g}^{x}(x \tau+u)\right]=0, \quad u \in \mathbb{R}^{n} .
$$

Therefore, by analogy with the case of entire functions (see [6, Chap. 6, Definitions 3.5 and 3.6]), we introduce the following concept.

Definition 3.4. For each fixed $u \in \mathbb{R}^{n}$, the nonvertical line

$$
E=\left\{\left(x \tau+u, \tau+\delta_{g}^{x}(u)\right) \in \mathbb{R}^{n} \times \mathbb{R}: \tau \in \mathbb{R}\right\}
$$

is called the (upper one-dimensional) $x$-asymptote of the function $W_{g}$, and the cylinder $I_{g}(x)=\operatorname{epi} \delta_{g}^{x}$ whose boundary (the graph of the function $y=W_{g}(u), u \in \mathbb{R}^{n}$ ) is the ruled surface formed by all $x$-asymptotes of the function $W_{g}$, is called an asymptotic $x$-cylinder of the epigraph epi $W_{g}$ of the function $W_{g}$.

We note an important nontrivial property of the $x$-type function for functions of the class $H\left(\mathbb{T}^{n}\right)$, which contains the criterion of finite type function in a given direction of growth and its structure. 
We denote by $Y_{H}=\{\rho\}$ the class of all finite, nonnegative, sublinear functions in $\mathbb{R}^{n}$ except for the function $\rho_{0}(u) \equiv 0, u \in \mathbb{R}^{n}$. Let $\rho \in Y_{H}$ and

$$
\mathfrak{M}_{n}(\rho)=\left\{g \in H\left(\mathbb{T}^{n}\right): \rho_{g}(u) \equiv \rho(u), u \in \mathbb{R}^{n}\right\}
$$

where $\rho_{g}$ is the order function for $g$ (see $(11)$ ); $\mathfrak{M}_{n}(\rho)$ is a subclass of $H\left(\mathbb{T}^{n}\right)$ with a given order function (or with a given order hypersurface, see (17)) $T^{\rho}=\left\{u \in \mathbb{R}^{n}: \rho(u)=1\right\}$ ). Consider the class

$$
\mathfrak{N}_{n}^{x}(\rho)=\left\{g \in \mathfrak{M}_{n}(\rho): 0<\sigma_{g}(x)<\infty\right\},
$$

where $x \in T^{\rho}$ and $\sigma_{g}(x)$ is the type of $g$ in the direction $x$ (see (17)). With a function $\rho \in Y_{H}$, we associate the convex compact set

$$
K_{\rho}=\left\{y \in \mathbb{R}^{n}:\langle y, u\rangle \leq \rho(u) \forall u \in \mathbb{R}^{n}\right\},
$$

whose support function is $\rho$. Let

$$
\partial \rho(x)=\left\{y \in K_{\rho}:\langle y, x\rangle=\rho(x)\right\}
$$

be the face of $K_{\rho}$ orthogonal to the vector $x \in T^{\rho}$ and

$$
\partial \rho(x)=\left\{y \in \mathbb{R}^{n}: \rho(u) \geq \rho(x)+\langle u-x, y\rangle \forall u \in \mathbb{R}^{n}\right\}
$$

be the subdifferential of the convex function $\rho$ at the point $x$ (see [11, Chap. 5, Sec. 23]). Based on the method of the proof of Theorem 3.9 in [6], we obtain the following assertion in the notation of the formulas (18)-(23).

Theorem 3.2 (see [6]). Assume that $g \in \mathfrak{N}_{n}^{x}(\rho)$, where $\rho \in Y_{H}$ and $x \in T^{\rho}$ (see (21), (17)). With a function $\rho \in Y_{H}$, we associate a convex compact set. Then the $x$-type function $\sigma_{g}(\cdot ; x)$ of the function $g$ possesses the following properties:

(1) $\sigma_{g}\left(r t^{x} ; x\right) \equiv t \sigma_{g}(r ; x)$ for all $t \geq 0, r \in \mathbb{R}_{0}^{n}$;

(2) there exists a unique convex, lower semicontinuous function $\psi: \partial \rho(x) \rightarrow(-\infty, \infty], \psi \not \equiv \infty$, such that the following representation is valid:

$$
\sigma_{g}(r ; x)=\sup \left\{r_{1}^{y_{1}} \ldots r_{n}^{y_{n}} \exp \{-\psi(y)\}: y \in \partial \rho(x)\right\}, \quad r \in \mathbb{R}_{0}^{n} .
$$

In particular, if the function $\rho \in Y_{H}$ is differentiable at a point $x \in T^{\rho}$, then there exists a constant $A_{g}=A_{g}(x)>0$ with the property

$$
\sigma_{g}(r ; x)=A_{g} \cdot \prod_{i=1}^{n} r_{i}^{\partial \rho / \partial x_{i}}, \quad r \in \mathbb{R}_{0}^{n} .
$$

Remark 3.1. We give another formulation of Theorem 3.2, which will be useful below.

We denote by $N_{n}^{x}(\rho)$ the class of positive functions $\varphi$ in $\mathbb{R}_{0}^{n}$ that are logarithmically convex ${ }^{2}$ with respect to $\ln r_{1}, \ldots, \ln r_{n}$ and possess the properties (1) and (2) of $x$-type functions specified in Theorem 3.2; here $\rho \in Y_{H}$ and $x \in T^{\rho}$. In this notation,

$$
\left\{\sigma_{g}(\cdot ; x), g \in \mathfrak{N}_{n}^{x}(\rho)\right\} \subset N_{n}^{x}(\rho) .
$$

Let us clarify the geometric meaning of Theorem 3.2 assuming, for simplicity, as in Theorem 3.1, that $W_{g}$ is a convex function (see the remark to Theorem 3.9 in [6]). From (24), using the notation of Proposition 3.3, we obtain

$$
\delta_{g}^{x}(u)=\ln \sigma_{g}\left(e^{u} ; x\right)=\sup \{\langle u, y\rangle-\psi(y), y \in \partial \rho(x)\}, \quad u \in \mathbb{R}^{n},
$$

\footnotetext{
${ }^{2}$ That is, the function $W_{\varphi}(u)=\ln \varphi\left(e^{u}\right), u \in \mathbb{R}^{n}$, is convex for all $\varphi \in N_{n}^{x}(\rho)$.
} 
where $\langle u, y\rangle$ is the scalar product in $\mathbb{R}^{n}$. However,

$$
\psi(y)=\left(\delta_{g}^{x}\right)^{*}(y)=\sup \left\{\langle u, y\rangle-\delta_{g}^{x}(u), u \in \mathbb{R}^{n}\right\}, \quad y \in \mathbb{R}^{n}, \quad\left\{y \in \mathbb{R}^{n}:\left(\delta_{g}^{x}\right)^{*}(y)<\infty\right\} \subset \partial \rho(x)
$$

is the Young transformation of the function $\delta_{g}^{x}$ (see [11, Chap. 3], [6, Chap. 1, Proposition 5.3 and Corollary 5.4]). Therefore, each supporting hyperplane to the asymptotic $x$-cylinder $I_{g}(x)=\operatorname{epi} \delta_{g}^{x}$ of the epigraph epi $W_{g}$ of the function $W_{g}$ (see (12)) is parallel to some supporting hyperplane to the asymptotic cone $A\left(\right.$ epi $\left.W_{g}\right)=$ epi $\rho_{g}$ of the convex set epi $W_{g}$ passing through the ray $\{(x t, t) \in$ $\left.\mathbb{R}^{n} \times \mathbb{R}, t>0\right\}$ (see Definitions 3.4 and 3.2 and explanations to them).

In the case of the class $\mathcal{A}\left(\mathbb{T}^{n}\right)$ (see Definition 2.2), there are simple formulas for the relationship between characteristics of the growth of functions of this class and their corresponding equivalent entire functions. In a similar form, they are available in [9, Theorem 2].

Proposition 3.4. Let $g \in \mathcal{A}\left(\mathbb{T}^{n}\right) ; \rho_{g}$ be a finite order function for the function $g$, $f$ be an entire function equivalent to $g, B=\left\|s_{k j}\right\|$ be an integer nondegenerate $(n \times n)$-matrix whose elements are the exponents of a monomial mapping $\mathcal{F}$ such that $f=[g \circ \mathcal{F}]($ see $(6))$. We set $\mathcal{B}_{p}=\pi_{p} \circ B^{-1}$, where

$$
p \leq n, \quad \pi_{p}: \mathbb{R}^{n} \rightarrow \mathbb{R}^{p}, \quad \pi_{p}\left(v_{1}, \ldots, v_{n}\right)=\left(v_{1}, \ldots, v_{p}\right), \quad \mathcal{E}_{p}=\pi_{p} \circ E^{-1},
$$

and $E$ is the trace of the mapping $\mathcal{F}$ to $\mathbb{R}_{0}^{n}$ (see (8)).

1. We have

$$
\rho_{f}\left[\mathcal{B}_{p}(u)\right] \equiv \rho_{g}(u), \quad u \in \mathbb{R}^{n},
$$

where $\rho_{f}$ is a function of orders for $f$.

2. If, in addition, for fixed $x \in \mathbb{R}^{n} \backslash\{0\}$, the conditions $0<\rho_{g}(x)<\infty$ and $0<\sigma_{g}(x)<\infty$ are satisfied, where $\sigma_{g}(x)$ is the $x$-type of $g$, then the type functions for $g$ and $f$ in the directions $x$ and $y=\mathcal{B}_{p}(x)$, respectively, are related by the formula

$$
\sigma_{f}(q ; y) \equiv \sigma_{g}(r ; x), \quad q=\mathcal{E}_{p}(r), \quad r \in \mathbb{R}_{0}^{n} .
$$

Proof. We use the notation from the proof of Corollary 2.1. The assertion 1 follows from the formulas (9) and Definition 3.1 (see (11)). Here we take into account the fact that the function $V_{f}(v):=\ln M_{f}\left(e^{v}\right), v \in \mathbb{R}^{n}$, is independent of the variables $v_{p+1}, \ldots, v_{n}$ for $p<n$. (Recall that $M_{f}$ is the maximum modulus of the entire function $f$ on the skeleton of the polydisk.)

Since by the assumption $\rho_{g}$ is a finite function, $0<\rho_{g}(x)<\infty$, and $0<\sigma_{g}(x)<\infty$, by Theorem 3.2, the type function $\sigma_{g}(\cdot ; x)$ is also finite. The assertion 2 is a consequence of the equality

$$
M_{f}(q)=\left[M_{g} \circ E\right](q), \quad q \in \mathbb{R}_{0}^{n},
$$

and Definition 3.3 (see (18)), if we again take into account the fact that the function $M_{f}(q)$ is independent of the variables $q_{p+1}, \ldots, q_{n}$ for $p<n$ (see Definition 2.2).

4. Characteristics of the growth of functions of the class $H\left(\mathbb{T}^{n}\right)$ and the coefficients of their expansion in the Laurent series. This section is devoted to deriving formulas that establish a connection between growth characteristics of functions from the class $H\left(\mathbb{T}^{n}\right), n>1$, and the coefficients of their Laurent expansions. These formulas are a complete analog of the corresponding results for the class $H\left(\mathbb{C}^{n}\right)$ of entire functions (see [6, Chap. 7, Theorem 1.4]), but their proofs are more complicated.

For each function $g \in H\left(\mathbb{T}^{n}\right)$, the following $n$-fold Laurent expansion converging everywhere in $\mathbb{T}^{n}$ holds: (see $[14$, p.40]):

$$
g(z)=\sum_{k \in \mathbb{Z}^{n}} a_{k} z^{k}, \quad z^{k}=z_{1}^{k_{1}} \ldots z_{n}^{k_{n}}, \quad a_{k}=\frac{1}{(2 \pi i)^{n}} \int_{\Gamma_{r}} \frac{g(z) d z}{z^{k+I}}, \quad z^{k+I}=z_{1}^{k_{1}+1} \ldots z_{n}^{k_{n}+1} .
$$


Here $d z=d z_{1} \ldots d z_{n}, \Gamma_{r}=\left\{z \in \mathbb{T}^{n}:\left|z_{j}\right|=r_{j}, j=1, \ldots, n\right\}$ is the topological product of circles of radii $r_{j} \in(0, \infty), j=1, \ldots, n$. Further, we assume that the support of the Laurent series is an unbounded set in $\mathbb{Z}^{n}$, i.e., the function $g$ is not a Laurent polynomial.

The following statement is a convergence criterion of a multiple Laurent series everywhere in $\mathbb{T}^{n}$.

Theorem 4.1 (see [7] and [8, Theorem 3.4]). The n-fold Laurent series (26) is convergent everywhere in $\mathbb{T}^{n}$ if and only if its coefficients satisfy the condition

$$
\lim _{\|k\| \rightarrow \infty} \sqrt[\|k\|]{\left|a_{k}\right|}=0, \quad\|k\|=\sum_{j=1}^{n}\left|k_{j}\right| .
$$

We consider the following characteristic of the unbounded support $S_{g}$ of a function $g$.

Definition 4.1. Let $u \in \mathbb{R}^{n} \backslash\{0\}$ and $g \in H\left(\mathbb{T}^{n}\right)$. Consider the Laurent series (26) and the function $\lambda=\lambda_{u}(k)=\langle k, u\rangle, k \in S_{g}$, defined on the support $S_{g}$. The set $D_{g}$ of all vectors $\{u\}$ such that the function $\lambda_{u}$ is unbounded from above is called the growth cone of the support $S_{g}$.

The following assertion shows that the set $D_{g}$ is the cone of directions of growth which is more rapid than the power growth for the function $g$.

Proposition 4.1. Let $g \in H\left(\mathbb{T}^{n}\right)$. An element $x \in \mathbb{R}^{n} \backslash\{0\}$ (see the notation of Definition 4.1) does not belong to the cone $D_{g}$ if and only if for the maximum modulus of $g$ on the skeleton of the polydisk, the following estimate holds:

$$
M_{g}\left(r t^{x}\right) \leq C(r) t^{s}, \quad t \geq t_{0}>0 .
$$

Moreover, $\rho_{g}(x)=0, x \notin D_{g}$, where $\rho_{g}$ is the order function of the function $g$ (see Definition 3.1).

Proof. Necessity. As in the case of entire functions, the following inequality (see (26)) holds:

$$
M_{g}(r) \leq S_{g}(r):=\sum_{k \in S_{g}}\left|a_{k}\right| r^{k}, \quad r \in \mathbb{R}_{0}^{n} .
$$

Let $x \notin D_{g}, s<\infty$ be an upper bound of the function $\lambda_{x}(k)=\langle k, x\rangle, k \in S_{g}$. From the above inequality, for every fixed $r \in \mathbb{R}_{0}^{n}$ we obtain the estimate (28), in which $C=S_{g}(r)$.

Sufficiency. The complete analog of the Cauchy inequality for Taylor coefficients of an entire function is valid for the coefficients of the Laurent expansion of the function $g$ (see (26)):

$$
\left|a_{k}\right| r^{k} \leq M_{g}(r) \quad \forall r \in \mathbb{R}_{0}^{n}, \quad k \in S_{g} .
$$

Hence, if the condition (28) is satisfied for some $s \in \mathbb{R}$ and fixed $r \in \mathbb{R}_{0}^{n}$, we find

$$
\left|a_{k}\right| r^{k} t^{\lambda} \leq C(r) t^{s}, \quad \lambda=\langle k, x\rangle, \quad k \in S_{g}, \quad t \geq t_{0}>0 .
$$

This means that $\lambda=\lambda_{x}(k) \leq s, k \in S_{g}$, i.e., $x \notin D_{g}$.

The final statement of Proposition 4.1 follows from Definition 3.1 and Theorem 3.1.

Remark 4.1. If $s \leq 0$ (see the notation for the estimate (28)), then $x \in K_{V}$, where $K_{V}$ is the cone of decrease directions of the function $V_{g}(u)=\ln M_{g}\left(e^{u}\right), u \in \mathbb{R}^{n}$ (see (2) and Definition 2.1), i.e., $K_{V} \subset \mathbb{R}^{n} \backslash D_{g}$. (In the general case, $K_{V}=\varnothing$ is possible.)

Lemma 4.1. Let $g \in H\left(\mathbb{T}^{n}\right), x \in D_{g}$, where $x \in D_{g}$ is the growth cone of the support $S_{g}($ see Definition 4.1). Assume that for fixed $r \in \mathbb{R}_{0}^{n}$, there exist constants $\Delta$ and $A>0$ such that

$$
M_{g}\left(r t^{x}\right)<\exp \left\{A t^{\Delta}\right\} \quad \forall t>t_{0} .
$$

Then the coefficients of the Laurent series of the function $g$ (see (26)) satisfy the inequality

$$
\left|a_{k}\right| r^{k}<\left(\frac{A e \Delta}{\lambda}\right)^{\lambda / \Delta} \quad \forall \lambda>\lambda_{0} ; \quad \lambda=\langle k, x\rangle .
$$


Proof. From the Cauchy inequality for the coefficients of the Laurent expansion of the function $g$ (see the proof of Proposition 4.1) and the inequalities (29), we derive for sufficiently large $\lambda=\langle k, x\rangle$, taking into account the fact that $x \in D_{g}$, and setting $\tau=\ln t$ :

$$
-\ln \left(\left|a_{k}\right| r^{k}\right)>\sup _{\tau>\tau_{0}}\left[\lambda \tau-A e^{\Delta \tau}\right]=\frac{\lambda}{\Delta} \ln \frac{\lambda}{A e \Delta} .
$$

After elementary transformations, we obtain the inequality (30).

Next, we need an additional property of functions that are equivalent to entire functions (see Definition 2.2). Assume that $g \in \mathcal{A}\left(\mathbb{T}^{n}\right)$ and $f$ is an entire function such that $g \sim f$. With the function $g$ represented by the series (7), we associate the following holomorphic function $G$ whose Laurent expansion has the same support as the series (7):

$$
G(z)=\sum_{k \in S_{g}} z^{k}, \quad z^{k}=z_{1}^{k_{1}} \ldots z_{n}^{k_{n}} .
$$

This series is equivalent to a power series

$$
H(w)=[G \circ \mathcal{F}](w)=\sum_{m \in S_{f}} w^{m}, \quad w^{m}=w_{1}^{m_{1}} \ldots w_{p}^{m_{p}}, \quad p \leq n,
$$

whose support coincides with the support of the series representing the entire function $f$, since in the notation of Definition 2.2

$$
w^{m}=z^{k}, \quad c_{m}=a_{k}, \quad z=\mathcal{F}(w), \quad w \in \mathbb{T}^{n} ; \quad k \in S_{g}, \quad m=B^{\prime} k \in S_{f} \subset \mathbb{Z}_{+}^{p} \subset \mathbb{Z}_{+}^{n} .
$$

Here $\mathcal{F}=\mathcal{F}_{g}$ is a monomial mapping of the form (6), $\left\{c_{m}\right\}$ and $\left\{a_{k}\right\}$ are the Taylor coefficients of the function $f$ and the Laurent coefficients of the function $g$, respectively, and $B^{\prime}$ is the matrix transpose to $B=\left\|s_{k j}\right\|$ (see (6)). It is obvious that the power series $H(w)$ converges absolutely in $D_{p}=\left\{w \in \mathbb{T}^{p}: 0<\left|w_{i}\right|<1, i=1, \ldots, p\right\}$. Therefore, we can distinguish in the domain $D$ the convergence set of the series (31) located in the $\mathbb{R}_{0}^{n}$.

Proposition 4.2. In the above notation, the convergence domain of the series (31) contains the set

$$
\left\{b(\alpha):=E(\alpha)=E\left(\alpha_{1}, \ldots, \alpha_{n}\right) \in \mathbb{R}_{0}^{n}, \alpha \in(0,1)^{n}\right\},
$$

where $E$ is the trace of the mapping $\mathcal{F} \rightarrow \mathbb{R}_{0}^{n}($ see $(8))$, and $b(\alpha) \rightarrow I$ as $\alpha \rightarrow I$, where $I=(1, \ldots, 1)$.

Lemma 4.2. Let a function $g \in \mathcal{A}\left(\mathbb{T}^{n}\right), n>1$, be equivalent to an entire function $f, x \in D_{g}$ (see Definitions 2.2 and 4.1). Assume that the coefficients of the Laurent expansion (7) of the function $g$ satisfy the inequality (see (30))

$$
\left|a_{k}\right|\left(\frac{r}{b}\right)^{k}<\left(\frac{A e \Delta}{\lambda}\right)^{\lambda / \Delta} \quad \forall \lambda>\lambda_{0} ; \quad \frac{r}{b}=\frac{r_{1}}{b_{1}} \ldots \frac{r_{n}}{b_{n}} ; \quad \lambda=\langle k, x\rangle,
$$

for some $r \in \mathbb{R}_{0}^{n}, \alpha \in(0,1)^{n}, A>0, \Delta>0$, where (see the notation of Proposition 4.2) $b=b(\alpha)=$ $E(\alpha)$. Then there exists a constant $C$ such that

$$
M_{g}\left(r t^{x}\right)<C e^{A t^{\Delta}} \quad \forall t>t_{0} ; \quad r t^{x}=\left(r_{1} t^{x_{1}}, \ldots, r_{n} t^{x_{n}}\right) .
$$

Proof. We perform a change of variables in the inequality (33) as follows. We set $r=E(q)$, where $q \in \mathbb{R}_{0}^{n}$ (see (8)). Next, we transform the left-hand side of the inequality (33) using the formula (32):

$$
\left|a_{k}\right|\left(\frac{r}{b}\right)^{k}=\left|a_{k}\right|\left[\frac{E(q)}{E(\alpha)}\right]^{k}=\left|a_{k}\right|\left[E\left(\frac{q}{\alpha}\right)\right]^{k}=\left|c_{m}\right|\left(\frac{q}{\alpha}\right)^{m}, \quad m=B^{\prime} k, \quad k \in S_{g},
$$


where $\left\{c_{m}, m \in S_{f}\right\}$ are the Taylor coefficients of the entire function $f=g \circ \mathfrak{F}$ (see (6)). Now we transform the parameter $\lambda$ on the right-hand side of the inequality (33):

$$
\lambda=\langle k, x\rangle=\langle k, B y\rangle=\left\langle B^{\prime} k, y\right\rangle=\langle m, y\rangle, \quad k \in S_{g}, \quad m=B^{\prime} k \in S_{f},
$$

where $y:=\left(y_{1}, \ldots, y_{n}\right)=B^{-1} x \in \mathbb{R}_{0}^{n}, B=\left\|s_{k j}\right\|$ is a nondegenerate matrix that determine the monomial mapping $\mathcal{F}$ (see (6)), and $B^{\prime}$ is the transpose matrix for $B$. But for $p<n$ we obtain $S_{f} \subset \mathbb{Z}_{+}^{p}$, i.e., the coordinates $m_{p+1}, \ldots, m_{n}$ of the vector $m$ are equal to zero (see Definition 2.2 and the remark to it). Therefore, in the general case,

$$
\lambda=\langle k, x\rangle=\langle m, y\rangle_{p}:=\sum_{i=1}^{p} m_{i} y_{i}, \quad k \in S_{g}, \quad m=B^{\prime} k \in S_{f} .
$$

From this and from (35) we conclude that the inequality (33) is transformed to the following inequality for the Taylor coefficients of the function $f$ :

$$
\left|c_{m}\right|\left(\frac{q}{\alpha}\right)^{m}<\left(\frac{A e \Delta}{\lambda}\right)^{\lambda / \Delta} \quad \forall \lambda>\lambda_{0} ; \quad \lambda=\langle m, y\rangle_{p} .
$$

From the equality

$$
M_{f}\left(q t^{y}\right)=M_{g}\left(r t^{x}\right), \quad r=E(q) \quad t>0,
$$

taking into account the fact that the function $M_{f}(v)=M_{g} \circ E(v), v \in \mathbb{R}_{0}^{n}$, is independent of the variables $v_{p+1}, \ldots, v_{n}$ for $p<n$ (see Definition 2.2), we conclude that $\left(y_{1}, \ldots, y_{p}\right) \in D_{f}$, where $D_{f}$ is the growth cone of the support $S_{f}$ of the function $f \in H\left(\mathbb{C}^{p}\right)$, since $x \in D_{g}$.

Under these conditions, there exists a constant $C>0$ such that the entire function $f$ satisfies the inequality

$$
M_{f}\left(q t^{y}\right)<C e^{A t^{\Delta}} \quad \forall t>t_{0} ; \quad q t^{y}=\left(q_{1} t^{y_{1}}, \ldots, q_{p} t^{y_{p}}\right)
$$

(see [6, Chap. 7, Lemma 1.3]). Returning to the former variable, we verify the validity of the lemma (see $(36)-(37))$.

Now we derive the relations between growth characteristics of functions of the class $H\left(\mathbb{T}^{n}\right), n>1$, and the coefficients of their Laurent expansions.

Theorem 4.2 (see [6, Chap. 7, Theorem 1.4]). Let $g \in H\left(\mathbb{T}^{n}\right), D_{g}$ be the growth cone of the support $S_{g}$ (see Definition 4.1). We assume that

$$
\beta_{g}(u)=\limsup _{\lambda \rightarrow \infty} \frac{\lambda \ln \lambda}{-\ln \left|a_{k}\right|}, \quad \lambda=\langle k, u\rangle ; \quad u \in D_{g},
$$

where $\left\{a_{k}\right\}$ are the coefficients of the Laurent expansion of the function $g($ see $(26))$, and $\left(-\ln \left|a_{k}\right|\right)^{-1}=$ 0 , if $a_{k}=0$. Then the following assertions hold.

(1) The order function $g$ (see Definition 3.1) possesses the property $\rho_{g}(u) \equiv \beta_{g}(u), u \in D_{g}$.

(2) If $\rho \in Y_{H}, g \in \mathfrak{M}_{n}(\rho)$ (see the notation of the formulas (20)-(21)), then for every $x \in\{u \in$ $\left.\mathbb{R}^{n}: \rho(u)>0\right\}$, the type function $\sigma_{g}(r ; x)$ of the function $g$ (see Definition 3.3) is defined by the formula

$$
\left[\sigma_{f}(r ; x) e \rho(x)\right]^{1 / \rho(x)}=\limsup _{\lambda \rightarrow \infty} \lambda^{1 / \rho(x)} \cdot\left(\left|a_{k}\right| r^{k}\right)^{1 / \lambda}, \quad \lambda=\langle k, x\rangle, \quad \rho(x)=\rho_{g}(x), \quad r \in \mathbb{R}_{0}^{n} .
$$

Proof. 1. Fix $x \in D_{g}$. If $\rho_{g}(x)<\infty$, then the inequality (29) is valid for $A=1, r=\mathbb{I}$, and $\Delta=\rho_{g}(x)+\varepsilon$, where $\varepsilon>0$. Under these restrictions, based on Lemma 4.1, we conclude that the inequality (30) is valid. Hence, after elementary transformations we obtain (see (38))

$$
\beta_{g}(x) \leq \rho_{g}(x)+\varepsilon \quad \forall \varepsilon>0,
$$


i.e., $\beta_{g}(x) \leq \rho_{g}(x)$. Note that $\beta_{g}(u) \geq 0, u \in D_{g}$, since $\ln \left|a_{k}\right| \rightarrow-\infty$ as $\lambda \rightarrow \infty$ (see (38) and (27)). So, $\beta_{g}(x)=\rho_{g}(x)$, if $\rho_{g}(x)=0$.

2. Now we assume that $\rho_{g}(x)>0$. According to Theorem 2.6 for the function $g \in H\left(\mathbb{T}^{n}\right)$, we conclude the formula (10) is valid. We consider the subset $\left\{g_{j} \in \mathcal{A}\left(\mathbb{T}^{n}\right), j \in A_{x}\right\}$ of terms that "form" the function $g$, where

$$
A_{x}=\left\{i \in(1, \ldots, m): S\left(g_{i}\right) \cap \Pi_{x} \neq \varnothing\right\}, \quad \Pi_{x}=\left\{k \in \mathbb{Z}^{n}: \lambda=\langle k, x\rangle>\lambda_{0}\right\}
$$

for any sufficiently large values of $\lambda_{0}>0$. We ensure that $A_{x} \neq \varnothing$. Otherwise, there is a number $\lambda_{0}$ such that $\langle k, x\rangle \leq \lambda_{0}$ for all $k \in S_{g}$, where $S_{g}$ is the support of the function $g$. This means that $x \notin D_{g} ;$ a contradiction.

Assume that $\beta_{g}(x)<\infty$ (see (38)). We denote by $\beta_{i}(x)$ a number that differs from $\beta_{g}(x)$ only by the rule that we set $k \in S\left(g_{i}\right)$ in (38) for $u=x$, where $S\left(g_{i}\right)$ is the support of the term $g_{i} \in \mathcal{A}\left(\mathbb{T}^{n}\right)$ of the function $g$ (see (40)). Let $\mathcal{F}_{i}$ be a monomial mapping of the form (6), existing for the function $g_{i}$, $E_{i}$ be the trace of the mapping $\mathcal{F}_{i}$ on $\mathbb{R}_{0}^{n}$, and $b^{(i)}=b^{(i)}(\alpha)=E_{i}(\alpha), \alpha \in(0,1)^{n}$ (see Definition 2.2 and Proposition 4.1). Theorem 4.1 implies the relation (see (38))

$$
\beta_{i}(x)=\limsup _{\lambda \rightarrow \infty} \frac{\lambda(\ln \lambda-\ln e \Delta)}{-\ln \left|a_{k}\right|+\left\langle k, \ln b^{(i)}\right\rangle} \leq \Delta, \quad \lambda=\langle k, x\rangle, \quad k \in S\left(g_{i}\right) ; \quad i \in A_{x} ; \quad \Delta=\beta_{g}(x)+\varepsilon,
$$

where $\varepsilon>0$. Therefore, the estimate for the Laurent coefficients of the term $g_{i}$ of the function $g$ is

$$
\left|a_{k}\right|<\left[b^{(i)}\right]^{k}\left(\frac{e \Delta}{\lambda}\right)^{\lambda / \Delta} \quad \forall \lambda>\lambda_{0} ; \quad \lambda=\langle k, x\rangle, \quad k \in S\left(g_{i}\right), \quad i \in A_{x} .
$$

Hence, applying Lemma 4.2, we arrive at the following inequality for $A=1, r=\mathbb{I}, \Delta=\beta_{g}(x)+\varepsilon$, and $\varepsilon>0$ :

$$
M_{g_{i}}\left(t^{x}\right)<C e^{t^{\Delta}} \quad \forall t>t_{i} ; \quad i \in A_{x}
$$

Finally, from the formula (10) we deduce (see the notation of the relations (40))

$$
M_{g}(r) \leq \sum_{i=1}^{m} M_{g_{i}}(r)=\Sigma_{1}+\Sigma_{2}, \quad \Sigma_{1}=\sum_{i \in A_{x}} M_{g_{i}}(r), \quad \Sigma_{2}=\sum_{i \notin A_{x}} M_{g_{i}}(r), \quad r \in \mathbb{R}_{0}^{n} .
$$

Now, based on the inequality (41) and Proposition 4.1, we conclude that there are constants $C_{j}>0$, $j=1,2$, and $s \in \mathbb{R}$ such that

$$
M_{g}\left(t^{x}\right)<C_{1} e^{t^{\Delta}}+C_{2} t^{s}, \quad t>0 .
$$

Applying the formula (11), we obtain

$$
\rho_{g}(x) \leq \Delta=\beta_{g}(x)+\varepsilon, \quad \rho_{g}(x) \leq \beta_{g}(x) .
$$

The opposite inequality was proved above. Moreover, we have proved that the assumption on the finiteness of one of the numbers $\rho_{g}(x)$ or $\beta_{g}(x)$ implies the finiteness of the other. Therefore, the equality $\rho_{g}(x)=\beta_{g}(x)$ is also valid in the case where one of these numbers is $\infty$. So, the formula (38) is valid.

3. The assertion (2) of the theorem is proved in the same way. When using Lemmas 4.1 and 4.2 we assume that $\Delta=\rho(x)=\rho_{g}(x)$. According to Theorem 3.2 , the $x$-type function $\sigma_{g}(\cdot ; x)$ is finite. This determines the finiteness of the function $A_{g}(r ; x), r \in \mathbb{R}_{0}^{n}$, defined by the right-hand part of the formula (39), which is convex with respect to $\ln r_{1}, \ldots, \ln r_{n}$. In particular, if $g \in \mathcal{A}\left(\mathbb{T}^{n}\right)$, then, applying Proposition 4.2, we find the equality

$$
A_{g}(r ; x)=\lim _{\alpha \rightarrow I} A_{g}\left[\frac{r}{b(\alpha)} ; x\right], \quad I=(1, \ldots, 1), \quad r \in \mathbb{R}_{0}^{n},
$$

used in the proof of the formula (39). 
Remark 4.2. Under the condition $g \in \mathcal{A}\left(\mathbb{T}^{n}\right)$, the proof of the theorem is substantially simplified: it is based on similar formulas for entire functions (see [6, Chap. 7, Theorem 1.4]). This can be verified by applying the method of change of variables used in the proof of Lemma 4.2 (see also [9]).

\section{REFERENCES}

1. W. Fulton, Introduction to Toric Varieties, Princeton Univ. Press, Princeton, New Jersey (1993).

2. A. G. Khovansky, "Newton polytopes (solution of singularities)," Itogi Nauki Tekhn. Sovr. Probl. Mat. Nov. Dostizh., 22, 207-239, VINITI, Moscow (1983).

3. P. Lelong and L. Gruman, Entire Functions of Several Complex Variables, Springer-Verlag, Berlin (1986).

4. L. S. Maergoiz, "Function of orders and scales of growth of entire functions of many variables," Sib. Mat. Zh., 13, No. 1, 118-132 (1972).

5. L. S. Maergoiz, "Functions of types of an entire function of several variables in the directions of its growth," Sib. Mat. Zh., 14, No. 5, 1037-1056 (1973).

6. L. S. Maergoiz, Asymptotic Characteristics of Entire Functions and Their Applications in Mathematics and Biophysics, Kluwer Academic, Dordrecht-Boston-London (2003).

7. L. S. Maergoiz, "Multidimensional analog of Laurent expansion of holomorphic functions and related questions," Dokl. Ross. Akad. Nauk, 452, No. 5, 486-489 (2013).

8. L. S. Maergoiz, "Extensions of the class of entire functions of several variables and related topics," Sib. Mat. Zh., 55, No. 5, 1137-1159 (2014).

9. L. S. Maergoiz, "Laplace-Borel transformation of functions holomorphic in the torus and equivalent to entire functions," in: Methods of Fourier Analysis and Approximation Theory, Birkhäuser, Basel (2016), pp. 195-209.

10. D. A. Raikov, Vector Spaces [in Russian], Fizmatgiz, Moscow (1962).

11. P. T. Rockafellar, Convex Analysis, Princeton Univ. Press, Princeton, New Jersey (1970).

12. L. I. Ronkin, Introduction to the Theory of Entire Functions of Many Variables [in Russian], Nauka, Moscow (1971).

13. L. I. Ronkin, "Entire functions," Itogi Nauki Tekhn. Sovr. Probl. Mat. Fundam. Napr., 9, 5-36, VINITI, Moscow (1986).

14. B. V. Shabat, Introduction to Complex Analysis, Part II, Nauka, Moscow (1985).

M. N. Zavyalov

Siberian Federal University, Krasnoyarsk, Russia

E-mail: zavyalovmn@mail.ru

L. S. Maergoiz

Federal Research Center "Krasnoyarsk Science Center of the Siberian Branch

of the Russian Academy of Sciences," Krasnoyarsk, Russia

E-mail: bear.lion@mail.ru 